\title{
A neural network model for dose-response of foodborne pathogens
}

\author{
Simon X. Yang* \\ Advanced Robotics and Intelligent Systems (ARIS) Lab, School of Engineering, University of Guelph, Guelph, Ont., Canada N1G 2 W1
}

Received 6 December 2001; received in revised form 1 November 2002; accepted 16 December 2002

\begin{abstract}
Foodborne infections are a significant cause of morbidity and mortality in human populations. Risk assessment and public health control measures could be greatly enhanced by establishing an accurate relationship between ingested dose and infection probability, and defining minimum infectious doses. In this paper, a novel neural network model is proposed for dose-response of foodborne pathogens. The proposed model assumes a three-layer structure with a fast back-propagation learning algorithm. The model predictions for four available datasets from the literature are compared using six statistical models: log-normal, log-logistic, simple exponential, flexible exponential, Beta-Poisson and Weibull-Gamma. The methods of least square error, maximum likelihood and correlation coefficient are used for the comparison study that shows the neural network model does better than the statistic models. Predictions of dose-response for multiple types of pathogens and dose-response with different host age and gender using neural network models are discussed with simulations.
\end{abstract}

(C) 2003 Elsevier B.V. All rights reserved.

Keywords: Neural network; Dose-response; Foodborne pathogens; Quantitative risk assessment

\section{Introduction}

Foodborne illnesses are estimated to cause as many as 9000 deaths per year in US [9]. The cost of foodborne illness is estimated to exceed US\$ 5 billion per year in US, and US\$ 1.3 billion per year in Canada [3]. Growing public concern over the microbiological safety of food has prompted the government and industry to accept hazard analysis critical control point (HACCP) as the system to ensure food safety. This system is based on identifying the likelihood of transmitting foodborne pathogens along different points in the food chain and reducing, eliminating or control-

* Tel.: +1-519-8244120x52437; fax: +1-519-836-0227. E-mail address: syang@uoguelph.ca (S.X. Yang). ling hazards. There are many studies of quantitative risk assessment of foodborne pathogens $[2,5,8,13,18]$.

Dose-response modeling of microbial risks is a key step in quantitative risk assessment for foodborne pathogens, which provides a standard scale of infectious potential. To conduct quantitative risk assessment for foodborne pathogens, a suitable dose-response model is essential for estimating the probability of infection (or illness) resulting from a certain level of exposure. The mathematical relationship between the ingested dose and the probability of infection (or illness) can be applied to quantify the risk of infection by exposure to known number of bacterial cells. However, the accurate dose-response relation is difficult to describe for two reasons: (1) the variability in both host susceptibility and microorganism infectivity; and (2) the lack of experimental data $[6,9,16]$. 
Several statistical models have been used to describe microbial dose-response relation. Recently, Holcomb et al. [9] selected six statistical models in the literature: log-normal (LN), log-logistic (LL), simple exponential (SE), flexible exponential (FE), Beta-Poisson (BP) and Weibull-Gamma (WG) $[6,19,20,22]$, to describe the dose-response of foodborne pathogens. Holcomb et al. [9] suggested the LL and LN models are not suitable for dose-response of foodborne pathogens due to a rejected fit. The SE and FE models have been used in risk assessment for waterborne giardiasis [9]. The BP model is widely associated with microbial dose-response and has been used to model dose-response relation for several food- and waterborne pathogens. The WG model is derived by applying a Gamma distribution to the host-pathogen heterogeneity and is recommended as the best statistical model for dose-response relation of foodborne pathogens [9].

Neural networks are well known for their capacity to learn from real-life examples and to generalize the input-output relationship. Neural networks are used to solve a wide variety of problems in science and engineering, particularly for some areas where the conventional modeling methods fail $[24,25]$. A well-trained neural network model can be used as a predictive model for a specific application, which is a data processing system inspired by biological neural system. In this paper, a neural network model is developed to describe the dose-response relation for foodborne pathogens. Four datasets available from the literature, Shigella flexneri $(S f)$, Shigella dysenteriae $(S d)$, Campylobacter jejuni $(C j)$ and Salmonella typhosa $(S t)[4,9,21]$ are used to train the proposed neural network. The model predictions are compared with four suitable statistical models (SE, FE, BP and WG) that were applied to dose-response of foodborne pathogens by Holcomb et al. [9] in 1999. To the best of our knowledge, it is the first time that the dose-response of foodborne pathogens is investigated using neural networks.

This paper is organized as follows. Section 2 presents the methods for modeling the dose-response relation, including the statistical models and the proposed neural network model. The results using both the statistical models and the neural network model are presented in Section 3. Section 4 discusses several important issues in dose-response modeling, including infection probability of food with multiple pathogens, infection probability with the consideration of age and gender, the selection of neural network architecture, and the advantages and limitations of the proposed neural network model. Finally, a concluding remark is given in Section 5.

\section{Methods}

The commonly used methods for dose-response prediction of the foodborne pathogens are statistical models. In this paper, a novel method, a neural network model is developed to describe the dose-response relation. The available datasets for four foodborne pathogens, S. flexneri, S. dysenteriae, $C$. jejuni and $S$. typhosa [9], were obtained from feeding studies of foodborne pathogens to human volunteers. The subjects in these feeding studies were healthy adults. The datasets are listed in Table 1, where the dose is expressed in total number of colony forming units (cfu) ingested; "+/Total" represents the number

Table 1

Dose-response datasets from literature

\begin{tabular}{|c|c|c|c|c|c|c|c|}
\hline \multicolumn{2}{|c|}{$S f(2 \mathrm{a})$} & \multicolumn{2}{|c|}{$S d(\mathrm{~m} 131)$} & \multicolumn{2}{|c|}{$C j(\mathrm{a} 3249)$} & \multicolumn{2}{|c|}{ St (quailes) } \\
\hline Dose & $+/$ Total & Dose & $+/$ Total & Dose & $+/$ Total & Dose & $+/$ Total \\
\hline 180 & $6 / 36$ & 10 & $1 / 10$ & 800 & $5 / 10$ & $10^{3}$ & $0 / 14$ \\
\hline 5000 & $33 / 49$ & 200 & $2 / 4$ & 8000 & $6 / 10$ & $10^{5}$ & $32 / 116$ \\
\hline $10^{4}$ & $66 / 87$ & 2000 & $7 / 10$ & $9 \times 10^{4}$ & $11 / 13$ & $10^{7}$ & $16 / 32$ \\
\hline $10^{5}$ & $15 / 24$ & $10^{4}$ & $5 / 6$ & $8 \times 10^{5}$ & $8 / 11$ & $10^{9}$ & $40 / 42$ \\
\hline $10^{5}$ & $3 / 4$ & & & $10^{6}$ & $15 / 19$ & & \\
\hline $10^{6}$ & $7 / 8$ & & & $10^{8}$ & $5 / 5$ & & \\
\hline $10^{8}$ & $7 / 8$ & & & & & & \\
\hline
\end{tabular}


infected (+) and the total number exposed in feeding studies (Total), respectively. Table 1 also shows the dose-response information at low doses that is generally not available from feeding studies.

\subsection{Statistical models}

Holcomb et al. [9] selected six commonly used statistical models, LN, LL, SE, FE, BP and WG, and applied them to dose-response of foodborne pathogens. These models are used to fit each of the four datasets available using the maximum likelihood method. Goodness-of-fit is assessed by comparing the minimum $Y$ value (the definition of $Y$ is given in Section 4) to the appropriate tabulated $\chi^{2}$ value $[9,17]$. For a digested dose $d$, the probability of infection $P$ using the LN, LL, SE, FE, BP and WG models are given as follows.

The log-normal model is given as

$P=\phi\left(b_{0}+b_{1} \log _{10} d\right)$,

where $\phi$ is the cumulative normal distribution function, $b_{0}$ the intercept, and $b_{1}$ the $\log _{10} d$ slope parameter.

The log-logistic model is given as

$P=1+\left(\frac{1-p}{p}\right) \mathrm{e}^{-\varepsilon\left(\log _{10} d-\chi\right)}$,

where $\chi$ is the predicted dose at a specified value of $p$, the probability of infection; and $\varepsilon$ the curve rate value affecting spread of curve along dose axis.

The simple exponential is given as

$P=1-\mathrm{e}^{-k \log _{10} d}$,

where $k$ is the reflects host microorganism interaction probability, specifically, $k$ denotes fraction of microorganisms ingested that survive to initiate infection.

The flexible exponential model is given as

$P=1-p \mathrm{e}^{-\varepsilon\left(\log _{10} d-\chi_{1}\right)}$,

where $\chi_{1}$ is the predicted dose at a specified value of $(1-p)$; and $p$ and $\varepsilon$ the same as those in Eq. (2).

The Beta-Poisson model is derived from the simple exponential that assumes a Beta distribution for host-microorganism interaction instead of a constant. This model is given as

$P=1-\left(1+\frac{d}{\beta_{2}}\right)^{-\varepsilon_{2}}$, where $\varepsilon_{2}$ and $\beta_{2}$ are the parameters affecting shape of curve.

The Weibull-Gamma model is given as

$P=1-\left(1+\frac{d^{\chi_{3}}}{\beta_{3}}\right)^{-\varepsilon_{3}}$,

where $\varepsilon_{3}, \beta_{3}$ and $\chi_{3}$ are the parameters affecting shape of curve. Note that if $\chi=1$, then this model reduces to $\mathrm{BP}$ model; and if $\varepsilon_{3}=1$, then this model reduces to LL model.

The simulation and comparison studies by Holcomb et al. [9] suggested that only four models, SE, FE, BP and WG, are applicable to microbial dose-response modeling, while the LL and LN models are not suitable for dose-response of foodborne pathogens due to a rejected fit [9].

\subsection{Neural network model}

The neural network assumes a three-layer architecture. A typical neural network model is shown in Fig. 1. The output of the neural network has one neuron representing the probability of infection, while the ingested doses of different types of pathogens are the inputs to the neural network. Additional factors that also result in the probability of infection, such as age and gender, can also be incorporated into the neural network model, and used as the input parameters as well.

The connection weights between neurons are initialized randomly in the region of $[-1,1]$. For a typical three-layer neural network to model any nonlinear input-output relationship, the activation function of the hidden neurons must be a nonlinear function, otherwise the neural network is reduced to a single layer neural network $[1,11]$. The activation function of the output neurons can be a linear or nonlinear function. When a nonlinear function (e.g. sigmoid functions) is used as the activation function, the output is bounded to a finite interval (e.g. $(0,1)$ or $(-1,1)$ depending on the selection of sigmoid function), and a linear scalar function is needed at the output to normalize the target output. If a linear activation function is used, the output can be any value and no linear scalar function is needed. In this study, the activation function of the hidden neurons is a nonlinear sigmoid function defined as $y=\tanh (x)$, while the activation function of the output neuron is a linear function $y=x$ (simulations 


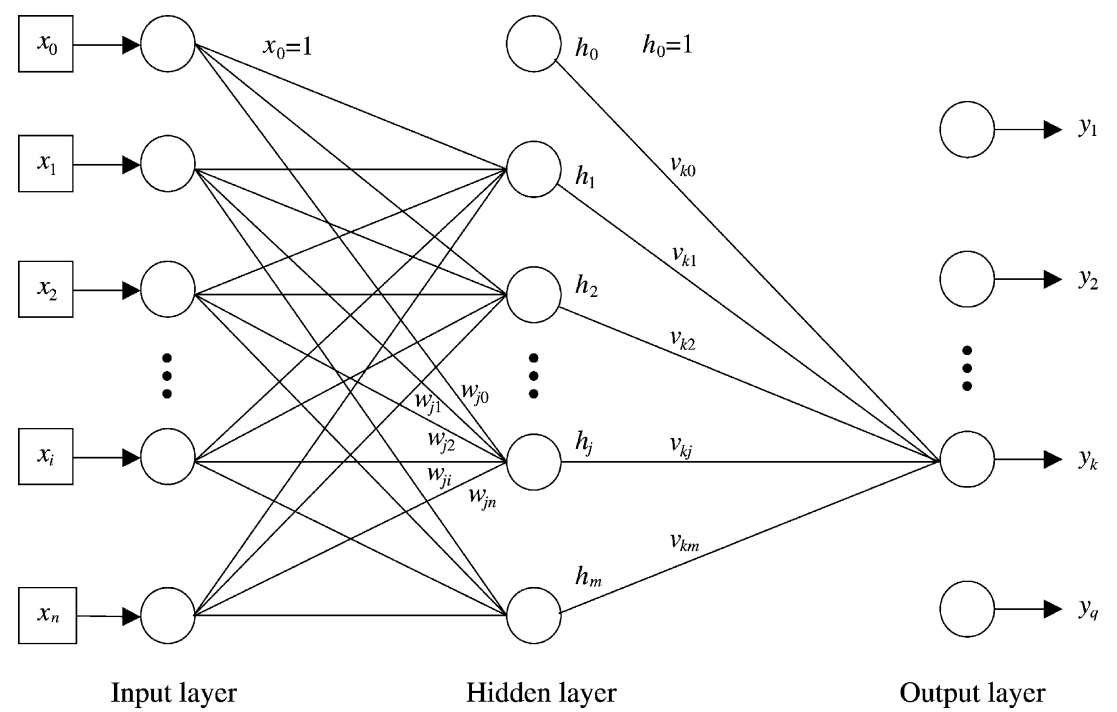

Fig. 1. A typical neural network structure.

show that a selection of a nonlinear activation $\tanh (x)$ at the output neuron results in the same results, but is more computationally complicated). Thus, the neural network output $y$ is given by

$y=\sum_{j=0}^{m} v_{j} h_{j}$,

where $h_{0} \equiv 1$, and variable $h_{j}$ is the output of the $j$ th hidden neuron, which is given by

$h_{j}=\tanh \left(\sum_{i=0}^{n} w_{j i} x_{i}\right)$,

where $x_{0} \equiv 1$, and $n$ and $m$ are the number of neurons in the input and hidden layers, respectively. Variable $x_{i}$ is the $i$ th input. Parameters $v$ and $w$ are the neural connection weights, which are normally initialized with a small random in the interval of $[-1,1]$ and will be set to a suitable value through a learning procedure with given data.

In this study, the fast algorithm proposed by Karayiannis and Venetsanopoulos [11,12] is used to train the neural network by minimizing an error function. The error function for neural network is normally defined as the squared errors, because this definition is easier for the derivation of the learning algorithm for the neural connection weights $[11,12]$. It would have a better fitting in log-likelihood if the error function is defined in log, but the learning algorithm will be much more difficult to obtain. Normally the error convergence will not highly depend on the method used. As shown in the comparison study in Fig. 6, where the model predictions are evaluated by squared errors $(E)$, correlation coefficient $(R)$ and maximum likelihood estimation $(Y)$, the predictions using the proposed neural network model are general better than all the statistical models, although the learning algorithm of the neural network is obtained by minimizing the squared errors $(E)$. Thus, in this study, the error function is defined as

$E=\sum_{k=1}^{s} e_{k}^{2}=\sum_{k=1}^{s}\left(t_{k}-y_{k}\right)^{2}$,

where $t_{k}$ and $y_{k}$ are the target value and neural network output for the $k$ th data sample, and $s$ the number of all the data samples. For a given input vector $x_{i}, i=$ $1,2, \ldots, n$, of a data sample, the learning algorithm modifying the connection weights is described by

$v_{j}=v_{j}+\alpha \varepsilon^{o} h_{j}, \quad j=0,1, \ldots, m$

$w_{j i}=w_{j i}+\alpha \varepsilon_{j}^{h} x_{i}$,

$i=0,1, \ldots, n, j=1, \ldots, m$

where

$\varepsilon^{o}=\lambda e+(1-\lambda) \tanh (\beta e)$, 

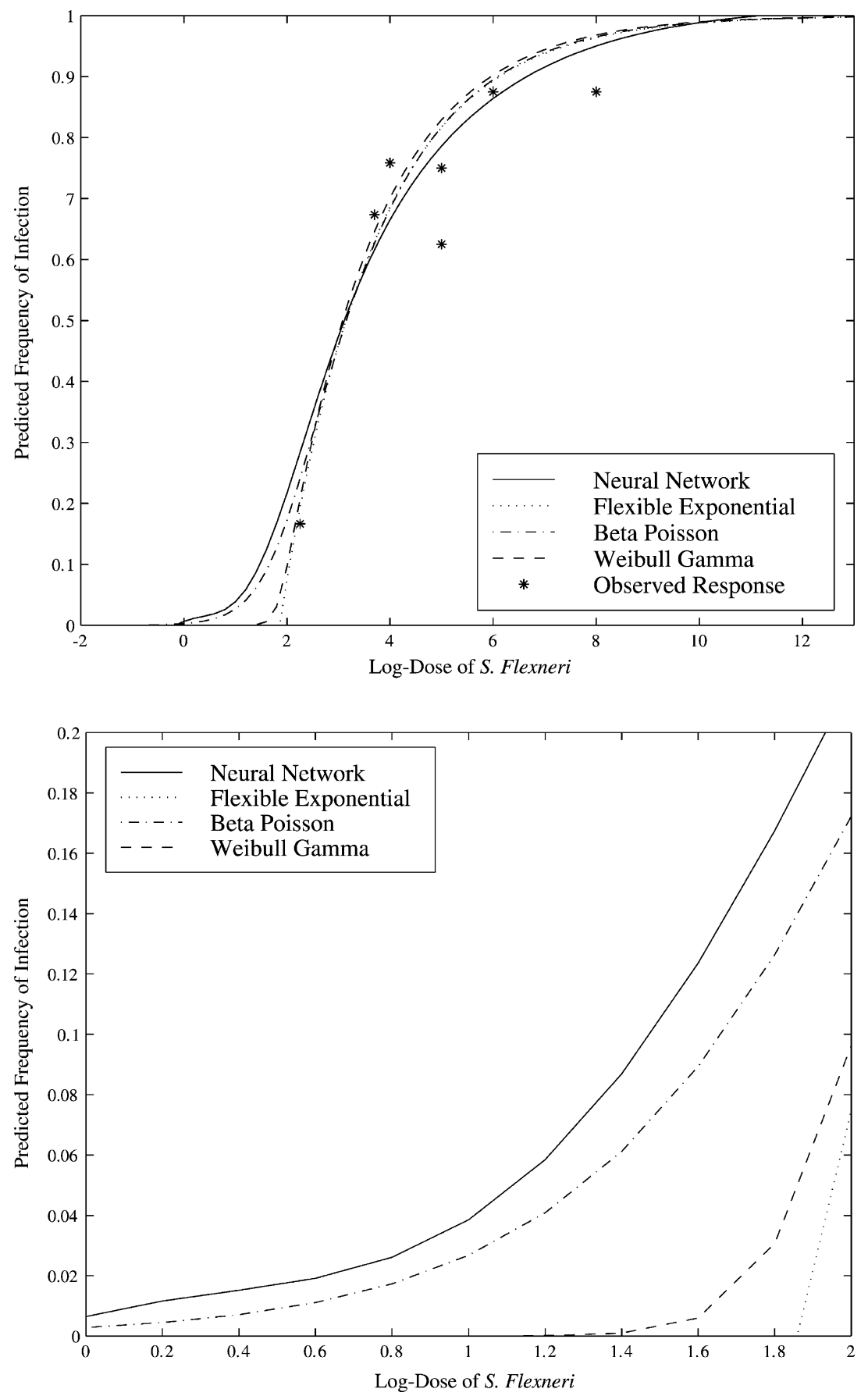

Fig. 2. Predicted frequency of infection vs. log-dose for S. flexneri using neural network model and three statistical models. The lower panel shows an enlarged view of the model predictions in the low-dose section. 
$\varepsilon_{j}^{h}=\left(1-h_{j}^{2}\right) \varepsilon^{o} v_{j}$,

$\lambda=\mathrm{e}^{-\mu / E^{2}}$.

where parameter $\alpha$ is the learning rate, and $\beta$ and $\mu$ the positive constants.

\section{Results}

The developed neural network model is applied to all the four available datasets. The performance of the neural network is compared to the four statistical models by least square error $(E)$ given in Eq. (9), correlation coefficient $(R)$ and maximum likelihood estimation $(Y)$. The loss function for maximum likelihood estimation $[7,9]$ is given by

$$
\begin{aligned}
Y=2 \sum_{i=1}^{n_{\mathrm{d}}}( & I_{i} \ln \left(\frac{I_{i}}{T_{i} P_{\text {inf }}}\right) \\
& \left.+\left(T_{i}-I_{i}\right) \ln \left(\frac{T_{i}-I_{i}}{T_{i}-T_{i} P_{\text {inf }}}\right)\right)
\end{aligned}
$$

where $n_{\mathrm{d}}$ is the number of the doses, $I_{i}$ the number of individuals infected at a dose level, $T_{i}$ the total number of individuals tested at a dose level, and $P_{\text {inf }}$ the predicted risk of infection. For a model prediction, with a smaller $Y$, the better; a smaller $E$, the better; the closer $R$ is to 1 , the better.

With the available datasets, the neural network architecture in Fig. 1 is used in our simulation studies. It is first applied to each dataset in Table 1. Thus, the neural network has one neuron in the input layer to represent the ingested dose, and one neuron in the output layer to represent the predicted frequency of infection. The simulations suggested that a selection of three neurons in the hidden layer is the best choice (for details, see Section 4). The predicted frequency of infection by $S$. flexneri is shown in the upper panel of Fig. 2 by a solid line. For comparison, the corresponding prediction using three statistical models with their best-fit parameters suggested by Holcomb et al. [9] are also plotted in Fig. 2. The SE model is not applicable for the data points of this pathogen [9]. It shows that the neural network prediction fits the data very well. The least square error $(E)$ for the seven

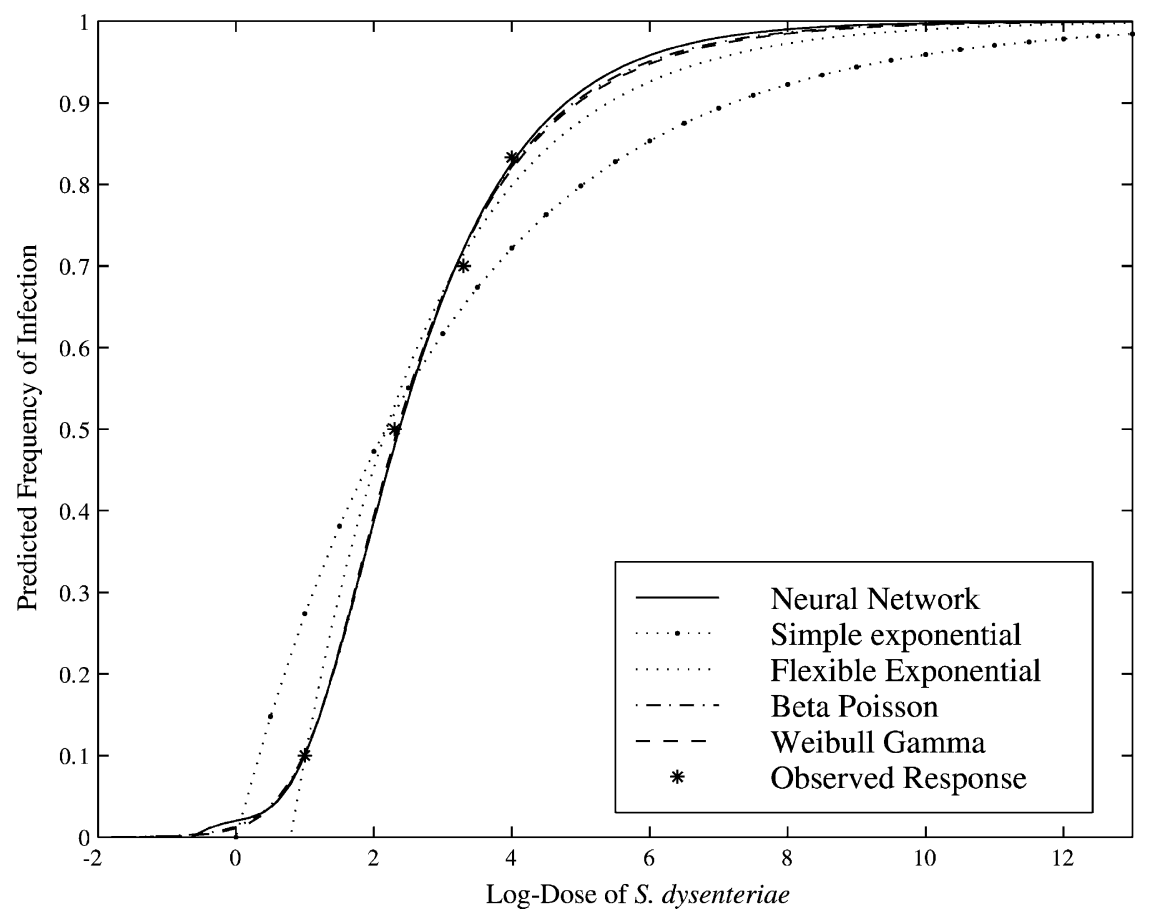

Fig. 3. Predicted frequency of infection vs. $\log$-dose for $S$. dysenteriae using neural network model and four statistical models. 


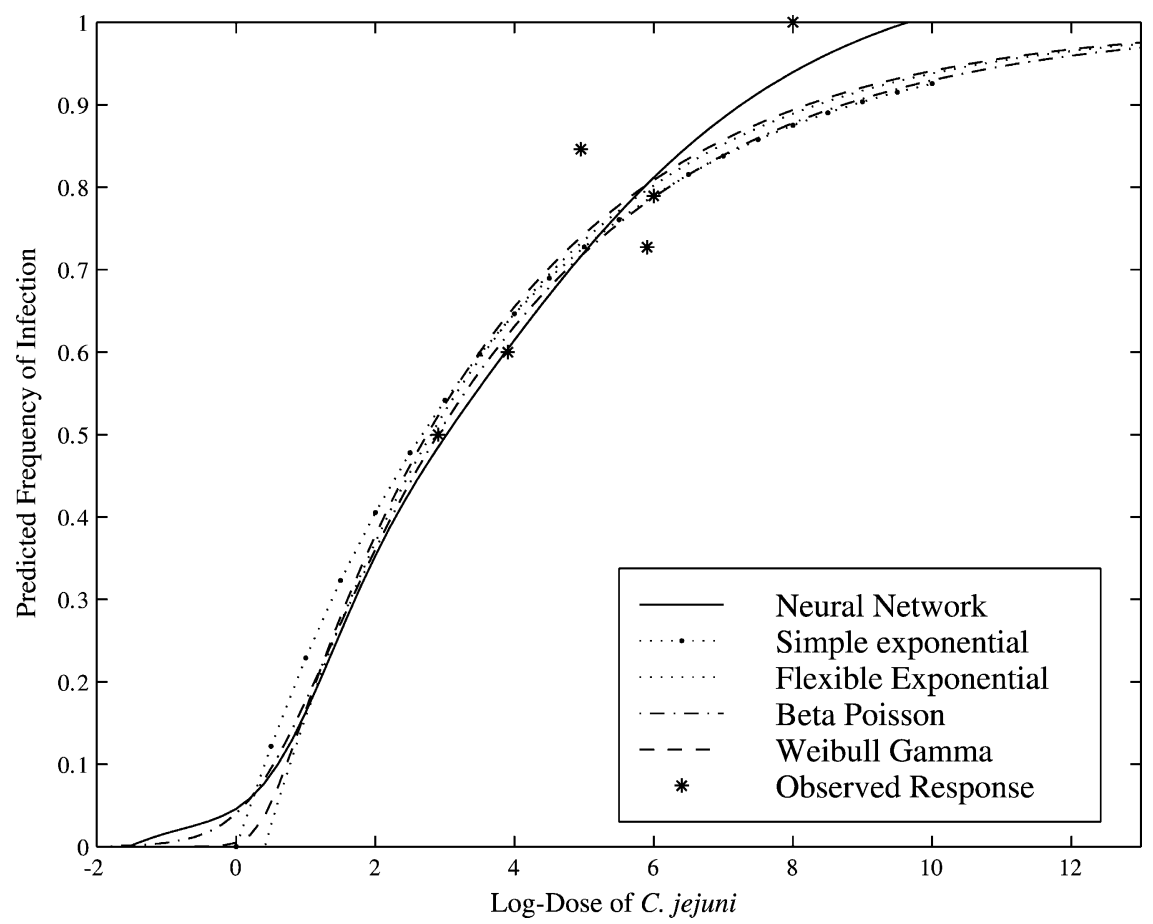

Fig. 4. Predicted frequency of infection vs. log-dose for $C$. jejuni using neural network model and four statistical models.

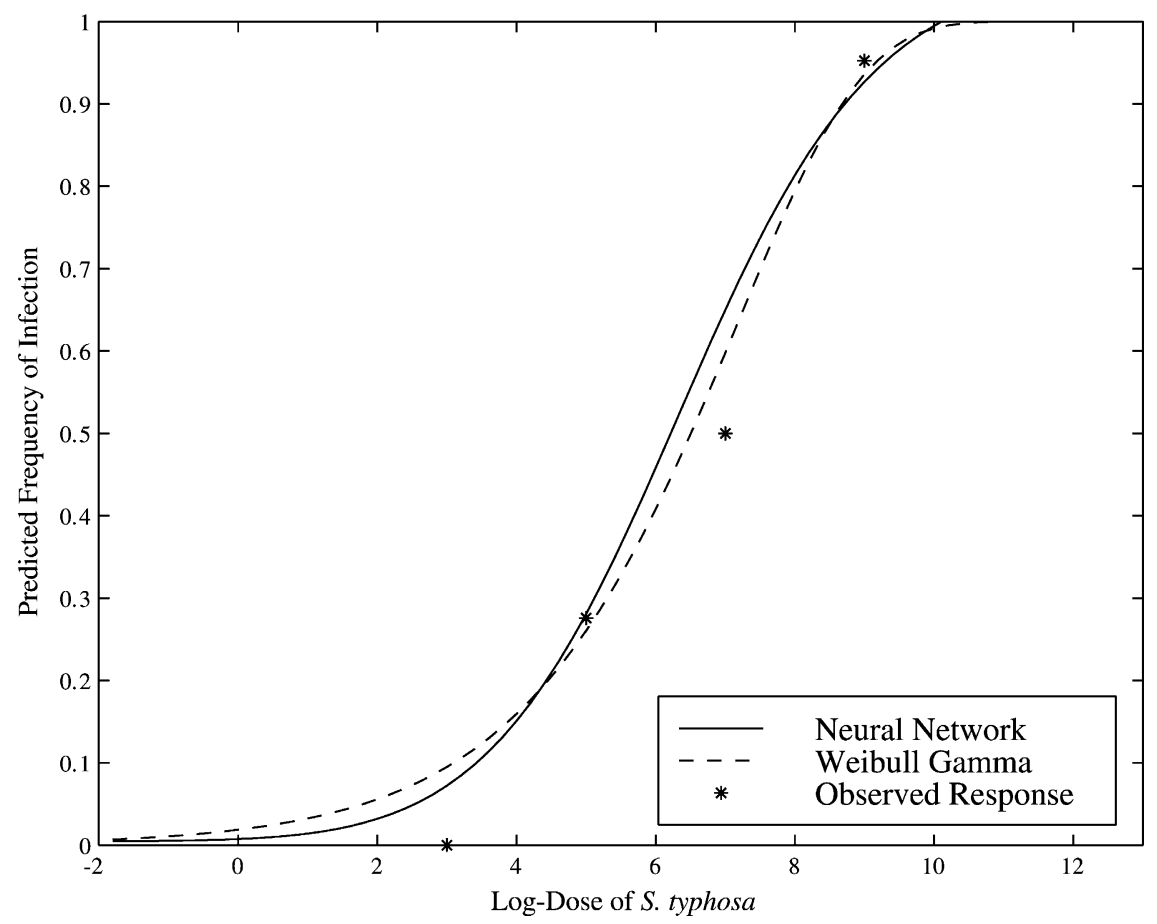

Fig. 5. Predicted frequency of infection vs. log-dose for S. typhosa using neural network model and WG model. 
data samples of $S$. flexneri is 0.059 . The correlation coefficient $(R)$ between the prediction and experimental data is 0.92 . Since there are no data available at the low-dose level, human knowledge is incorporated to get a reasonable prediction of infection frequency in the low-dose section, an enlarged view of which is shown in the lower panel of Fig. 2. Note that the response using the proposed neural network model is obviously higher than the statistical models. As there is no data to fit at the low-dose section, human knowledge is incorporated in the model training. The justification is that: (1) the response should not be zero at the low dose as some statistical models, such as FE model; and (2) a higher response means more precautious and safer. So, such an overestimating response is used in this paper to have a better protection of human health. When there are low-dose data available, future work should include those new data and consider probable network and plausible prediction by incorporating Bayesian methods and statistical framework $[14,15]$, e.g. using a hybrid model with neural work and statistical method [23].

The predicted frequency of infection by $S$. dysenteriae, C. jejuni and S. typhosa are shown in Figs. 3-5, respectively. Note that for $S$. typhosa, the WG model is the only statistical model which fit four data samples (see Fig. 5). It shows that the proposed neural network model is capable of describing well the dose-response relation of these pathogens. The least square errors (E) for S. dysenteriae, C. jejuni and S. typhosa are $0.001,0.027$ and 0.028 , respectively, while the correlation coefficients $(R)$ are $0.999,0.959$ and 0.998 , respectively.

In addition to the qualitative comparison among the neural network model and the four statistical models in Figs. 2-5, the performance of these models are also quantitatively compared using maximum likelihood estimation, least square error and correction efficient. The comparison among the neural network model and statistical models (SE, FE, BP and WG) for the four foodborne pathogens is shown in Fig. 6. The upper, middle and lower panels in Fig. 6 show the maximum likelihood estimate $(Y)$, least square error $(E)$, and correlation coefficient $(R)$, respectively. It shows that the SE model is not applicable to the data samples of $S$. flexneri and S. typhosa due to rejected fit [9]. The FE and BP models are not applicable to the data samples of $S$. typhosa. Therefore, WG is the only model
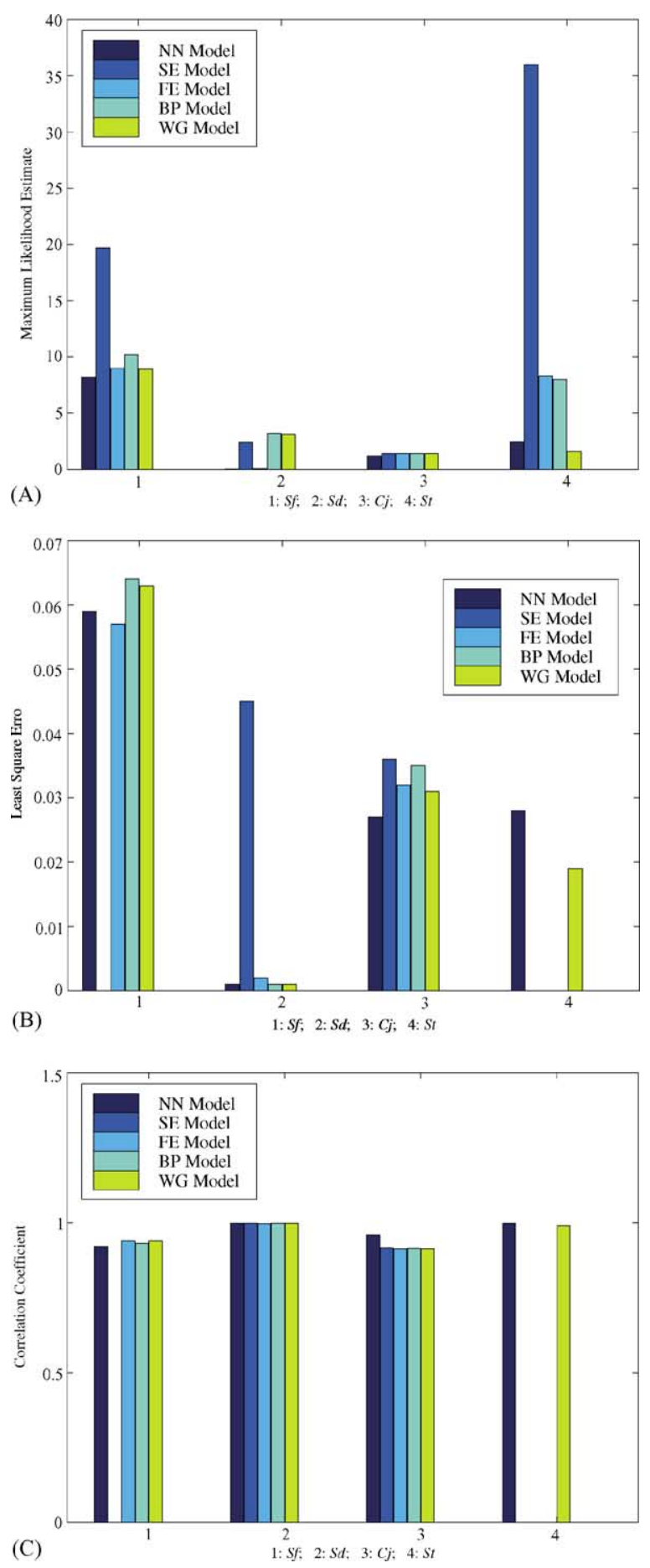

Fig. 6. The bar graphs for $Y, E$ and $R$ for each pathogen using neural network model and statistical models. 
capable of fitting all the data samples of all the four pathogens [9]. In comparison to the WG model, the performance of the proposed neural network is generally better.

\section{Discussion}

The proposed neural network model is capable of fitting all the datasets of four pathogens, generally better than the best statistical model, the WG model. In addition, the statistical models are unable to deal with the case of multiple pathogens. For those statistical models, different set of model parameters selected by trial and error has to be used to fit different types of pathogens using the same model. The proposed neural network is also theoretically capable of predicting the frequency of infection for a food containing more than one type of pathogens. After the neural network model is well trained, it deals with various types of pathogens without additional training procedures. The proposed unified neural network assumes four input neurons and nine hidden neurons. Since there are no data available for frequency of infection with multiple foodborne pathogens, the unified neural network model is applied to the four datasets in Table 1. The neural network prediction of infection frequency is shown in Fig. 7, where the model prediction is shown in solid line, while the experimental data are shown by dashed line. It shows that the unified neural network architecture is capable of fitting all the datasets from four types of pathogens. The correlation coefficients $(R)$ for S. flexneri, S. dysenteriae, C. jejuni and S. typhosa are $0.996,0.967,0.997$ and 0.947 , respectively.

Furthermore, the proposed model is capable of incorporating additional factors that influence the frequency of infection, such as age and gender of the host. A neural network is designed, with the same learning
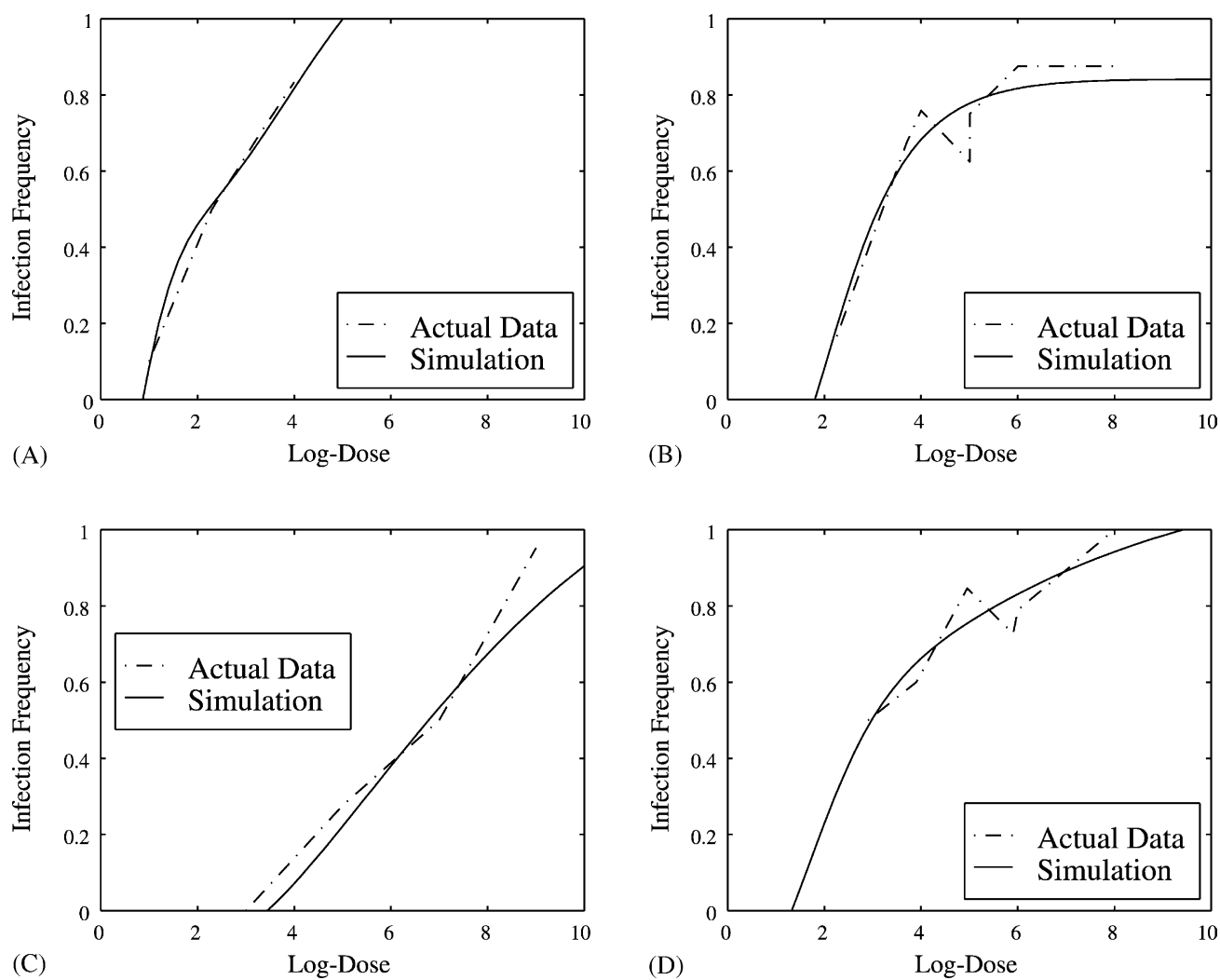

Fig. 7. Predicted frequency of infection vs. log-dose for all the four pathogens using the unified neural network model. 
algorithm presented in Section 2, for the prediction of inflection with the factors of dose, age and gender. Since there are limited data available, based on common knowledge of the age-related incident of infection, it is assumed for this study that the frequencies of infection of children and seniors are three and two times of the adults; the infection frequency of female is 1.2 times of the male (statistically the difference is not so significant), with the same ingested dose. Assume the dataset in Table 1 for $S$. dysenteriae is for adult male. The neural network has three input neurons that represents the dose, age (adult $=1$; senior $=2$; children $=3$ ), and gender (male $=1$; female $=2$ ). Three neurons are selected in the hidden layer. Model prediction of the inflection frequency with log-dose, age and gender is shown in Fig. 8. It shows that the model prediction is reasonably well.

Theoretically a three-layer neural network can model any nonlinear functions at any accuracy, provided enough number of neurons in the hidden layer and enough training/learning time [10,11]. However, the open questions in practical implementation are the selections of training algorithm and model parameters (e.g. number of hidden neurons, activation functions, learning rate, momentum, etc.) to guarantee the error to converge to a desired small number. When a three-layer neural network model is used to model the input-output relation of a specific problem, the number of input neurons in the input layer (also called first layer) is the number of inputs, while the number of output neurons in the last layer is the number of outputs. The number of hidden neurons can be selected with some flexibility, which determines how well a dataset can be learned. Too many hidden neurons will tend to memorize the problem, and thus do not generalize the input-output relationship. This is the so-called "over-fitting" problem. If the number of hidden neurons used is not enough, the network will generalize the relationship well but may not have enough "power" to learn the patterns well at a satisfactory accuracy. This the so-called "under-fitting" problem. Therefore, in practice the number of neurons in the hidden layer is mainly selected by trial and error, as there are some suggestions available, but no general methods available for an optimal selection of hidden neurons [10]. In this study, a series of simulations using the data of $S$. dysenteriae were conducted, where the hidden neurons were selected as 2, 3, 4 and 5, respectively.

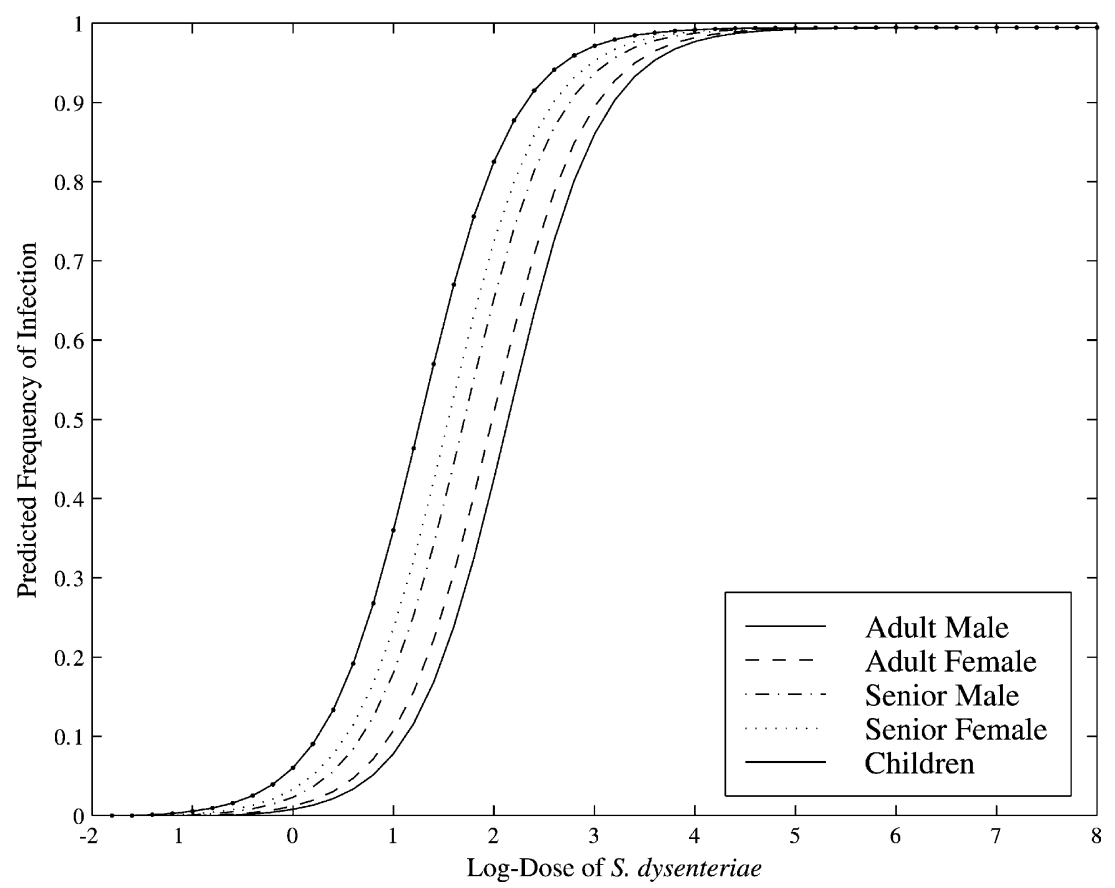

Fig. 8. Predicted frequency of infection vs. log-dose by $S$. dysenteriae for different ages and genders. 
The simulation results show that two hidden neurons cannot accurately fit the datasets, i.e. the under-fitting occurs. The datasets can be fitted satisfactory with three hidden neurons. When four and five hidden neurons are used, although the least square error $(E)$ is a little bit smaller, but the generated input-output curve is not as smooth as that with three hidden neurons, i.e. over-fitting occurs. So in this study, three hidden neurons are used for all available data.

When neural network is used to model an inputoutput relation, as any other modeling methods, it requires suitable data to train the neural network, such that it can learn the relationship. The lack of data is a common limitation for both the neural network model and the statistical models. Because of the ethical considerations, it is unlikely that adequate human data for dose-response will become available for highly infectious pathogens. An obvious alternative is the use of animal data. However, animal data must be carefully reviewed for the applicability to human, due to the inherent variability in host-microorganism interaction. Unlike the statistical models where all the model parameters are selected by trial and error, the neural network model is capable of automatically obtaining the neural connection weights through learning. However, the statistical models have less parameters to be set in comparison to the number of weights in the neural network model.

\section{Conclusion}

In this paper, a novel neural network model is proposed for dose-response of foodborne pathogens. The predictions using neural networks are better than the statistical models in the literature. In addition, it has good flexibility and can deal with dose-responses for more than one type of pathogen in a food. Furthermore, the proposed neural network model is capable of incorporating additional factors that influence the inflection frequency, such as age, in addition to the digested dose. Similar to the statistical models, the limitation and difficulty of the development of a better neural network is the lack of experimental data. The quality of a neural network model for dose-response highly depends on an understanding from the biological point of view and the experimental data available. The investigation on dose-response relationships us- ing neural networks not only provides an alternative, flexible, accurate model for prediction of inflection for various types of pathogens, but also offers insights into methodologies for quantitative risk assessment of foodborne pathogens.

\section{Acknowledgements}

This work was supported by Natural Sciences and Engineering Research Council (NSERC) of Canada. The author would like to thank Mr. Baoguo Xie and Mr. Guangfeng Yuan for their assistance in simulations and Dr. Mohamed Karmali and Dr. Anna M. Lammerding from their valuable comments.

\section{References}

[1] C.M. Bishop, Neural Networks for Pattern Recognition, Oxford University Press, Oxford, 1995.

[2] R.L. Buchanan, A.M. Lammerding, I.R. Clarke, T.A. Roberts, Potential application of risk assessment techniques to microbiological issues related to international trade in food and food products, J. Food Prot. 61 (8) (1998) 1075-1086.

[3] M.H. Cassin, A.M. Lammerding, E.C.D. Todd, W. Ross, R.S. McColl, Quantitative risk assessment for Escherichia coli O157:H7 in ground beef hamburgers, Intl. J. Food Microbiol. 41 (1998) 21-44.

[4] C.S. Crockett, C.N. Haas, A. Fazil, J.B. Rose, C.P. Gerba, Prevalence of Shigellosis in the US: consistency with doseresponse information, Intl. J. Food Microbiol. 30 (1996) 8799.

[5] M.E. Coleman, H.M. Marks, Qualitative and quantitative risk assessment, Food Control 10 (1999) 189-197.

[6] J.M. Farber, W.H. Rose, J. Harwig, Health risk assessment of Listeria monocytogenes in Canada, Intl. J. Food Microbiol. 30 (1996) 145-154.

[7] C.N. Haas, J.B. Rose, C. Gerna, S. Regli, Risk assessment of viruses in drinking water, Risk Anal. 13 (5) (1993) 545-552.

[8] C.N. Haas, J.B. Rose, C. Gerna, Quantitative Microbial Risk Assessment, Wiley, New York, 1999.

[9] D.L. Holcomb, M.A. Smith, G.O. Ware, Y.C. Hung, R.E. Brackett, M.P. Doyle, Comparison of six dose-response models for use with foodborne pathogen, Risk Anal. 19 (6) (1999) 1091-1100.

[10] S. Haykin, Neural Networks: A Comprehensive Foundation, Prentice-Hall, New Jersey, 1999.

[11] N.B. Karayiannis, A.N. Venetsanopoulos, Artificial Neural Networks, Kluwer Academic Publishers, Dordrecht, 1993.

[12] N.B. Karayiannis, A.N. Venetsanopoulos, Efficient learning algorithms for neural networks (ELEANNE), IEEE Trans. Syst. Man Cybern. 23 (5) (1993) 1372-1383.

[13] A.M. Lammerding, G.M. Paoli, Quantitative risk assessment: an emerging tool for emerging foodborne pathogens, Emerg. Infect. Dis. 3 (4) (1997) 438-487. 
[14] D.J.C. MacKay, Bayesian interpolation, Neural Comput. 4 (3) (1992) 415-447.

[15] D.J.C. MacKay, Probable networks and plausible predictions-a review of practical Bayesian methods for supervised neural networks, Netw. Comput. Neural Syst. 6 (3) (1995) 469-505.

[16] H.M. Marks, M.E. Coleman, C.T.J. Lin, T. Roberts, Topics in microbial risk assessment: dynamic flow tree process, Risk Anal. 18 (3) (1998) 309-328.

[17] P. McCullagh, J.A. Nelder, Generalized Linear Model, Chapman \& Hall, New York, 1989.

[18] W.B. Mcnab, A general framework illustrating an approach to quantitative microbial food safety risk assessment, J. Food Prot. 61 (9) (1998) 1216-1228.

[19] G.J. Medema, P.F.M. Teunis, A.H. Havelaar, C.N. Haas, Assessment of the dose-response relationship of Campylobacter jejuni, Intl. J. Food Microbiol. 30 (1996) 101111.
[20] S. Regli, J.B. Rose, C.N. Haas, C.P. Gerba, Modeling the risk from Giardia and viruses in drinking water, J. Am. Water Works Assoc. 83 (11) (1991) 76-84.

[21] J.B. Rose, C.N. Haas, S. Regli, Risk assessment and control of waterborne giardiasis, Am. J. Public Health 81 (6) (1991) 709-713.

[22] E.C.D. Todd, J. Harwig, Microbial risk analysis of food in Canada, J. Food Prot. (Suppl.) 59 (1996) 10-18.

[23] S.X. Yang, H. Li, J. Shi, Modeling of supercritical fluid extraction by neural networks, Intell. Autom. Soft Comput. 19 (1) (2003) 3-12.

[24] M.A. Zaidi, H. Murase, N. Honami, Neural network model for the evaluation of lettuce plant growth, J. Agric. Eng. Res. 74 (1999) 237-242.

[25] Q. Zhang, S.X. Yang, G.S. Mittal, S. Li, Prediction of performance indices and optimal parameters of rough rice drying using neural networks, Biosyst. Eng. 83 (3) (2002) 281-290. 